\title{
Dosimetric Analysis of Prone Breast Treatment in Tomotherapy and Conventional Linear Accelerator
}

\author{
Ching Chong Jack Yang ${ }^{1}$, Zhihui Hu${ }^{2}$, Yie Chen ${ }^{1}$, Jie Qiu ${ }^{3}$ \\ ${ }^{1}$ Monmouth Medical Center, Long Branch, USA \\ ${ }^{2}$ Chinese Academy Medical Science (CAMS), Beijing, China \\ ${ }^{3}$ Beijing Union Hospital, Beijing, China \\ Email: jyang@barnabashealth.org
}

Received August 19, 2013; revised September 21, 2013; accepted October 20, 2013

Copyright (C) 2013 Ching Chong Jack Yang et al. This is an open access article distributed under the Creative Commons Attribution License, which permits unrestricted use, distribution, and reproduction in any medium, provided the original work is properly cited.

\begin{abstract}
Purpose: To evaluate planning quality and dosimetric differences of clinically deliverable 3D conformal plans generated from Tomotherapy with TomoDirect ${ }^{\mathrm{TM}}$ (TD) and conventional field-in-field approach in prone breast treatment. Materials and methods: Total of twelve randomly selected early stage left breast patients who went through lumpectomy and were previously treated on traditional Linear Accelerator (LINAC) have been re-planned and tested on Tomotherapy TomoDirect module. Baseline prescription dose was chosen at 50.4 Gy $(1.8 \mathrm{~Gy} \times 28$ fractions $)$ to cover $\geq 95 \%$ of PTV for planning criteria with other critical structure dose constraints in the thoracic region. Planning outcomes such as $\mathrm{D}_{95}$ ( $95 \%$ of volume of PTV receiving the prescribed dose), $D_{5}$ and $D_{1}$, heart, both lungs as well as the contralateral breast were simultaneously evaluated. Conformity of the prescription isodose/volume to PTV was evaluated as conformity index (CI) and dose uniformity was also evaluated with homogeneity index (HI) in the same study series. All outcome parameters were analyzed and summarized to evaluate dosimetric impact of planning qualities between these two planning platforms. Results: The planning results indicate that $\mathrm{CI}, \mathrm{HI}, \mathrm{D}_{95}, \mathrm{D}_{5}$ and $\mathrm{D}_{1}$ of PTV, critical structures such as heart, ipsilateral and contralateral lungs as well as contralateral breast doses were comparable but with better overall statistical end points from TD plans. The $\mathrm{D}_{95}, \mathrm{D}_{5}$ and $\mathrm{D}_{1}$ of PTV for TD plans were superior in dosimetric analysis and more uniform than those plans generated from Pinnacle ${ }^{\mathrm{TM}}$ field-in-field planning technique. Overall, TD plans have superior planning quality than the conventional method does, with straightforward and automated planning process once the beam delivery parameters were established. Conclusions: From the clinical treatment planning results, plans from TD in general achieved better uniform tumor coverage with fewer hot spots while sparing more critical structures were based upon isodose distribution and Dose Volume Histogram (DVH) analysis. Image guidance of TD delivery automates the setup within the treatment bore without tedious verification process compared to the process with LINAC. Though all plans are deliverable, TD planning possesses dosimetric advantages due to its modulated optimization pattern. However, TD did present a challenge during the simulation if a patient is oversized with long pendulant breast which is hard to fit into the Tomotherapy ring structure. From our analysis, TD plans reserve superior dosimetric outcome with $\mathrm{CI}, \mathrm{HI}, \mathrm{D}_{95}, \mathrm{D}_{5}$, and $\mathrm{D}_{1}$ of PTV, and better sparing contralateral lung and breast doses.
\end{abstract}

Keywords: Field-In-Field; Tomo Direct; Prone Breast; Modulation

\section{Introduction}

Breast cancer has been a commonly discovered disease among US population. Radiation therapy plays a vital role in breast cancer management. With the radiation therapy, the mortality rate of breast cancer introduced by local recurrence is about $14 \%$ with 15 years follow-up [1]. Conventionally, parallel-opposed tangential beams are used to treat the whole breast and chest wall tissue with the supine position. Additional abutting megavoltage photon and electron fields might be added to treat the supraclavicular, axillary, and internal mammary lymph nodes for advanced stages of breast cancer. A typical complication for breast treatment is radiation induced cardiovascular side effects, and radiation-induced pneumontitis has been reported in approximately $30 \%$ of breast cancer patients treated with definitive radiotherapy [2-4]. Poor cosmesis or fibrosis of those larger breasts was also observed with different study groups with the stage I and II 
patients treated with lumpectomy and radiotherapy for curative intent $[5,6]$. One of the biggest challenges for traditional breast treatment setup lies in the immobilization with large or pendulant breast tissues and the daily setup has been a tedious process for the positional uncertainty. Those setup uncertainties may be a contributing factor for the complication that have been observed. Prone breast treatment was adopted for women with large and pendulous breasts, which achieve better dose uniformity with less hot spots, and better cosmetic and fewer toxicities [7,8].

Clinical evidence of 3-D conformal radiation therapy shows positive impact of intensive modification treatment with Multi-leaf Collimators (MLC) combination which can also produce uniform dose distribution compared to the wedge paired, opposing treatment fields. Planning principles of field-in-field concept have also been utilized with prone breast treatment in order to generate much more uniform dose distribution while avoiding the setup positional uncertainty on a daily basis with the supine, unsettled location [9-12]. With the TD module provided by Tomotherapy with the $3 \mathrm{D}$ treatment, fixed angle modality with constant couch movement, the Tomotherapy unit could also deliver static beam with the modulated fields of various MLC combinations. For patients who are not suitable for the supine position, prone breast treatment has been widely accepted as a protocol to reduce many unacceptable hot and cold spots and unwanted side effects such as dermatitis and possible cardiac perfusion defects [13-18]. LINAC based prone breast treatment encounters certain challenges such as collision possibility, setup uncertainty and most likely, the hot spots close to the border of the pendulant breast PTV volume which are dosimetrically challenging. Though Tomotherapy IMRT technique has been adopted to produce acceptable breast treatment, the low dose distribution to many other critical structures such as heart, contralateral lung and breast has been questionable for the gain of better dose uniformity coverage [19-21]. This study is to prove the benefits of utilizing TD in managing the prone breast treatment similar with the current fieldin-filed intensity modulated approach, with possibly more homogeneous PTV dose distributions.

TD is a discrete angle, and a non-rotational treatment delivery mode designed to complement the Tomotherapy IMRT delivery technique. It applies the principles by continuously translating the couch while using fewer fixed beams for treatment. TD delivers all beams for each target sequentially, with a single run of the couch movement to cover the full length of the breast with margins [22]. While still utilizing the beamlet-based delivery, it also allows users to plan and treat routine cases with fixed beam direction, in the same format of CT pilot scanning process with constant treatment couch movement.

\section{Materials and Methods}

Total of twelve patients diagnosed with early stage left breast cancer with lumpectomy treatment were randomly selected $(n=12)$ with prone breast delivery of 50.4 Gy prescription dose. Target volume and critical structure definition with PTV is limited to $5 \mathrm{~mm}$ from the skin surface to avoid hot spot spill to the skin tissue. Two skin structures were contoured, a $5 \mathrm{~mm}$ and a $2.5 \mathrm{~mm}$ strip extending from patient surface towards the breast PTV in TD planning. The volume of complete PTV structure ranged from $143.45 \mathrm{~cm}^{3}$ to $1541.29 \mathrm{~cm}^{3}$ with the mean volume of $647.80 \mathrm{~cm}^{3}$ (standard deviation [SD] \pm 391.07 $\mathrm{cm}^{3}$ ). All other critical structures such as lungs, heart, contra-lateral breast tissue, and unspecified tissue (for planning avoidance) were also delineated by the same physician. The unspecified tissue was defined as the body contours but excluding all the above delineated targets and critical structures in test cases.

Beam geometry (Opposing beam ports with pseudo half beam characteristics to avoid divergence at planning isocenter) was defined in each system (Tomo plan version 4.03 and Philips Pinnacle version 9.2). Since the TD planning is a non-uniform fluence delivery pattern with constant couch movement, binary MLC pattern has produced optimized MLC pattern with couch movement (pitch index) to generate conformal dose distribution, similar to the field-in-field technique. Optimization and calculation were performed in TD without further manual fine tuning; therefore, plan can be created within 15 minutes. LINAC based prone breast treatment is with fieldin-field segmental optimization method, with a purpose of producing homogeneous PTV coverage.

Planning criteria of the twelve selected left breast patients are based on the following instructions:

1) Both plans consists the same planning isocenter, while treatment delivery of TD is off centered;

2) Goals of planning are to at least $95 \%$ of the PTV with the prescription dose;

3) Minimize V107\% (Volume at $107 \%$ of the prescription dose) of the PTV coverage close to zero;

4) Philips Pinnacle Version 9.2: conventional 3D-CRT with field-in-field segmental optimization method. For each tangential field, 2 or 3 segments were used to homogenize the dose at the planning target volume (PTV);

5) Tomo plan version 4.2: TomoDirect 3D-CRT with two tangential beams. A field width of $2.5 \mathrm{~cm}$ and the default pitch of 0.25 were used to generate suitable projected DRR comparable to the field-in-field technique.

One major difference of the TD plan versus conventional 3D planning is that planning process is similar to the IMRT technique, dose constraints need to be entered to generate the optimized dosimetry results. Though the beam angles have to be specified for static beam entries, flash of the beam to cover the breast volume (usually 
around $2 \mathrm{~cm}$ ) is automatically added to provide enough field width for the beam ports. Couch movement is continuous; with the pitch and projected DRR patterns generated from MLC shapes does represent the modulated characteristics of prone beam delivery in Tomotherapy. Figure 1 has shown the typical beam arrangement and isocenter selection during the prone breast setup. For 3D CRT, patient markings were carefully selected at the rigid points which can be easily reproduced with SSD recordings (Figure 1(a)). In Tomotherapy setting, though there were no SSD readings, the relative shift in MVCT image verification helped to position the patient inside the gentry bore with lasers in image guidance (Figure 1(b)).

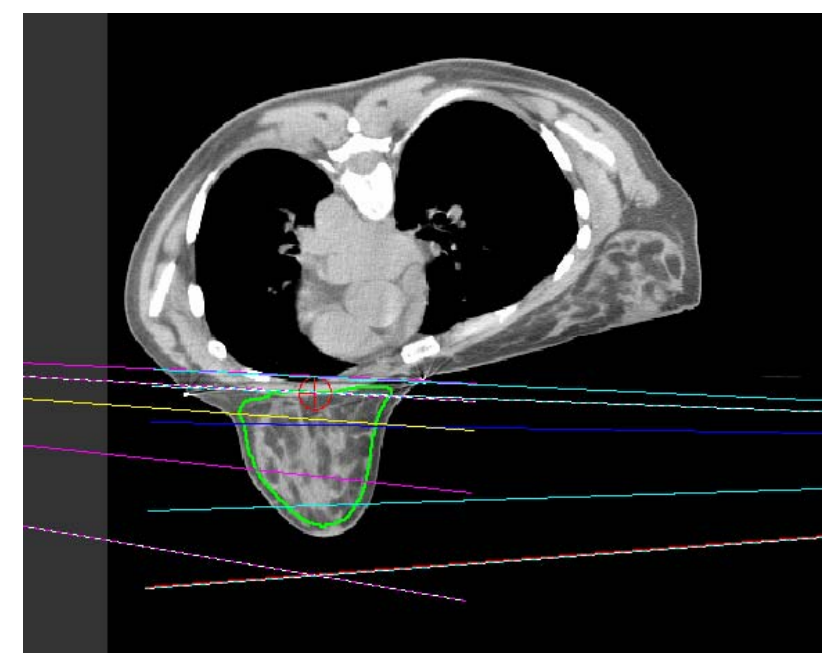

(a)

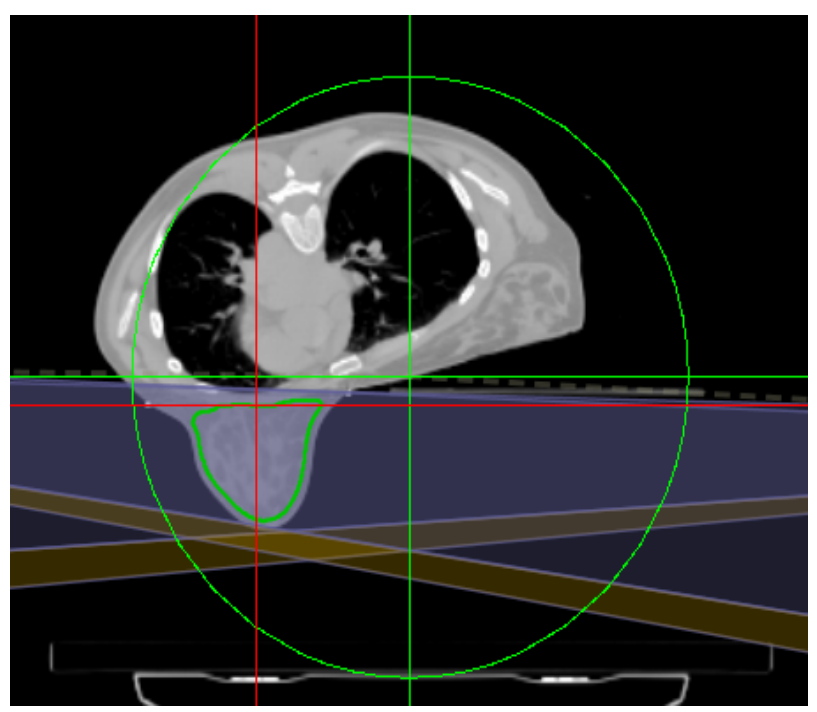

(b)

Figure 1. (a) Beam arrangement in conventional 3D-CRT with field-in-field, optimization methodology; (b) Beam arrangement in TomoDirect, the isocenter location is located at the center of the bore (green lines), and beam delivery center is off the machine isocenter (red lines).

\section{Results}

Planning dosimetry outcome has indicated superior dose conformity and better sparing of critical structures with the TD plans. Tomotherapy static beam delivery with the modulated MLC and couch movement combination can reduce the hot spots during the planning stage while making the setup easier compared to the conventional LINAC delivery methodology. One of the benefits for prone breast in Tomotherapy is that clearance has been confined inside the gantry ring structure with automatic couch movement function. Therefore, image guided pretreatment verification can be achieved with efficiency and collision possibility can be eliminated. However, with the ring gantry delivery, other dilemma is mechanical limitation for treating large sized breast with overweighed patients. During the pre-screening process in the patient simulation stage with our large bore CT simulator (large bore is $85 \mathrm{~cm}$ radius, the same size as Tomotherapy bore) certainly reduce the possibility of not being able to perform TD delivery if the patients can be setup and screened during this stage.

\subsection{Dose Distribution Analysis}

We observed that with the pre-defined prescription coverage ( $95 \%$ to the breast PTV), the hot spot of TD is less compared to the field-in-field conventional planning approach. Figure 2 indicates the typical isodose compari-

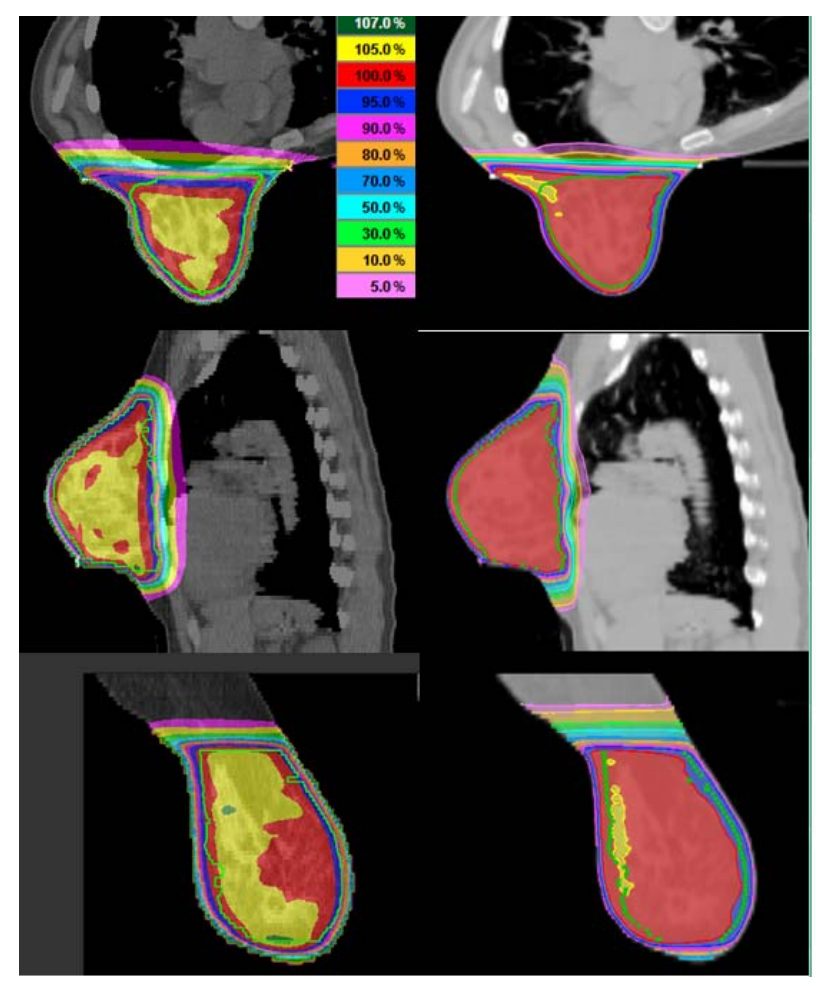

Figure 2. Dose displays in three orthogonal views (Left: Pinnacle; Right: TomoDirect). 
son between Pinnacle field-infield plans versus TD 3D plan. It is obvious that relationship in between these two delivery methodologies, a noticeable hot spot reduction is observed with TD. Exposures of ipsilateral lung and heart are also minimized, due to the hard constraint posted in the TD plan.

\subsection{DVH Analysis}

DVHs of PTV coverage and critical structure are also displayed from Figures 3(a)-(f), which indicate the planning advantages for TD, because of the automated proc- ess. The planning time for TD can be greatly reduced to within 15 minutes for all calculated dose criteria.

\subsection{Dosimetric Data Analysis}

Mean dose coverage of PTV for both TD and conventional field-infield plans are without statistically significance of a p-value of 0.893 . However, TD plan has better $\mathrm{D}_{95}$ and $\mathrm{D}_{99}$ coverage, while the hot spot of $\mathrm{D}_{1}$ is less than field-in-field 3D plan. The $\mathrm{V}_{100 \%}$ (volume for receiving the full prescription dose) is also higher in TD plan, with much homogeneous dose distributions. How-
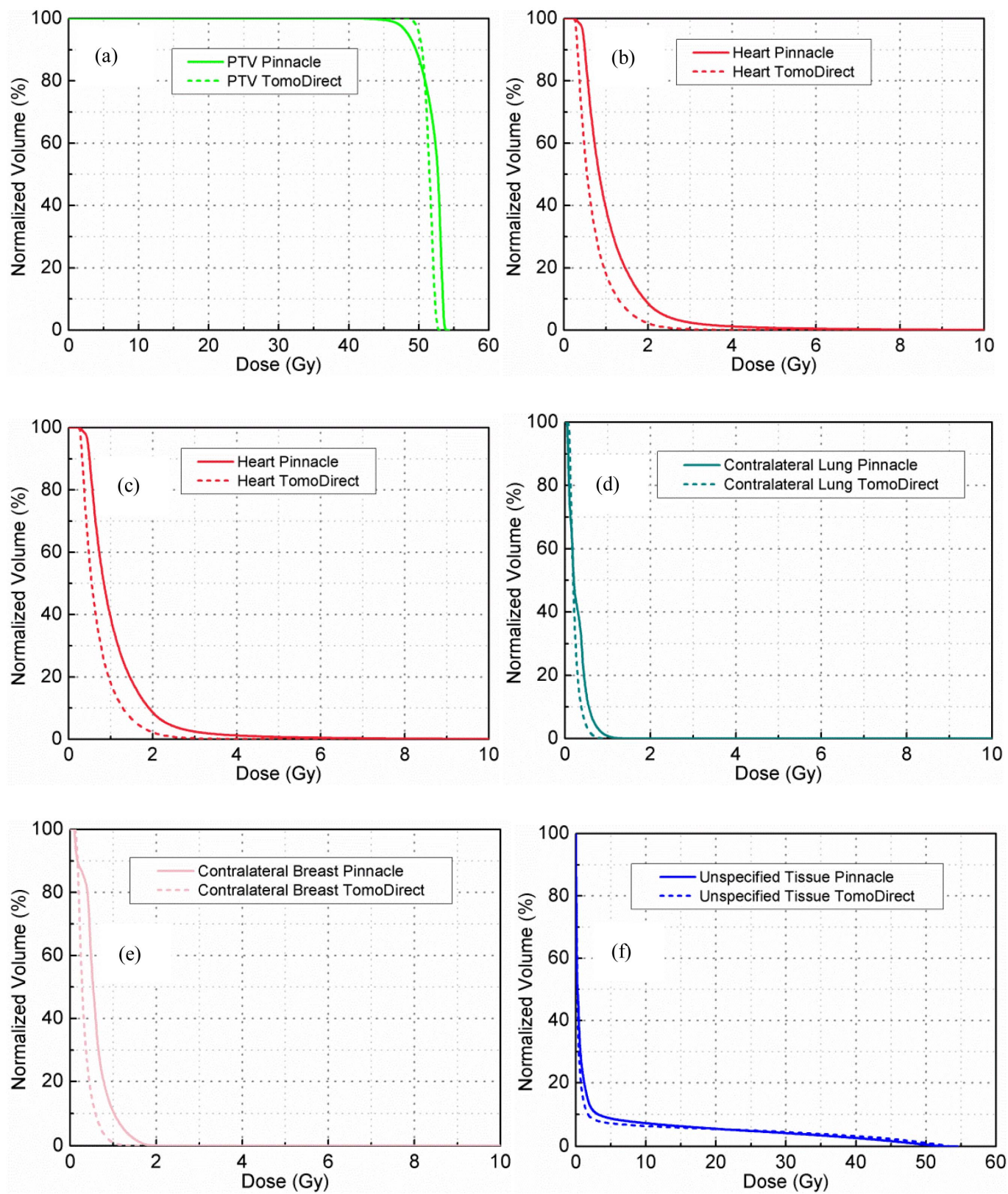

Figure 3. (a), (b) DVH comparisons of PTV and heart (solid-Pinnacle, dash-TD); (c), (d) DVH comparisons of ipsilateral and contra-lateral lungs (solid-Pinnacle, dash-TD); (e), (f) DVH comparisons of contralateral breast and external volumes (solidPinnacle, dash-TD). 
ever, conformal index (CI) of TD is less than optimum, which indicates TD plan has missed some PTV at the isocenter junction planes. Table 1 summarizes dose and volume planning results, also indicates the homogeneous distribution of TD inside the PTV, with superior $\mathrm{V}_{100 \%}$ coverage (Table $\mathbf{1})$.

In Table 2, all dosimetric data related to the quality of plans have been reported. The $1 \mathrm{cc}$ heart and ipsilateral doses are significant lower in TD plan compared to the
3D field-infield approach. Dose constraints addressed in TD has been optimized while at the same time, PTVcoverage is maintained with comparable coverage. One of the key factors for the heart and lung sparing difference is that the 3D field-in-field plan in Pinnacle still relies on planner's personal skill in selecting the treatment areas or critical structures to achieve dose uniformity, therefore, the maximum heart and ipsilateral doses in planning process may be neglected in these cases.

Table 1. Target volume coverage.

\begin{tabular}{|c|c|c|c|c|c|}
\hline \multirow{2}{*}{ PTV } & \multicolumn{2}{|c|}{ Conventional 3D-CRT (field-in-field) } & \multicolumn{2}{|c|}{ TomoDirect 3D-CRT } & \multirow{2}{*}{$p$ Value $^{\mathrm{a}}$} \\
\hline & Range & Mean \pm SD & Range & Mean \pm SD & \\
\hline Mean Dose (Gy) & $51.07-52.04$ & $51.52 \pm 0.29$ & $51.13-51.73$ & $51.53 \pm 0.17$ & 0.893 \\
\hline $\mathrm{D}_{1}(\mathrm{~Gy})$ & $53.00-53.88$ & $53.53 \pm 0.29$ & $52.11-53.16$ & $52.71 \pm 0.29$ & 0.000 \\
\hline $\mathrm{D}_{95}(\mathrm{~Gy})$ & $47.98-48.61$ & $48.28 \pm 0.18$ & $50.06-50.58$ & $50.39 \pm 0.16$ & 0.000 \\
\hline $\mathrm{D}_{99}(\mathrm{~Gy})$ & $45.88-47.24$ & $46.40 \pm 0.38$ & $49.01-50.08$ & $49.76 \pm 0.38$ & 0.000 \\
\hline $\mathrm{V}_{90 \%}(\%)$ & $99.3 \%-99.9 \%$ & $99.58 \% \pm 0.20 \%$ & $99.9 \%-100.0 \%$ & $99.98 \% \pm 0.03 \%$ & 0.000 \\
\hline $\mathrm{V}_{95 \%}(\%)$ & $95.3 \%-97.7 \%$ & $96.34 \% \pm 0.60 \%$ & $99.7 \%-100.0 \%$ & $99.93 \% \pm 0.08 \%$ & 0.000 \\
\hline $\mathrm{V}_{100 \%}(\%)$ & $74.9 \%-84.9 \%$ & $80.70 \% \pm 3.10 \%$ & $91.0 \%-97.0 \%$ & $94.98 \% \pm 1.84 \%$ & 0.000 \\
\hline $\mathrm{V}_{105 \%}(\%)$ & $1.5 \%-41.7 \%$ & $16.38 \% \pm 12.43 \%$ & $0.0 \%-2.9 \%$ & $0.74 \% \pm 0.95 \%$ & 0.001 \\
\hline $\mathrm{V}_{107 \%}(\%)$ & $0.0 \%-0.7 \%$ & $0.18 \% \pm 0.27 \%$ & $0.0 \%-0.1 \%$ & $0.02 \% \pm 0.04 \%$ & 0.081 \\
\hline $\mathrm{HI}^{\mathrm{b}}$ & $1.09-1.12$ & $1.10 \pm 0.01$ & $1.02-1.05$ & $1.04 \pm 0.01$ & 0.000 \\
\hline $\mathrm{CI}^{\mathrm{c}}$ & $0.66-0.79$ & $0.75 \pm 0.04$ & $0.74-0.87$ & $0.81 \pm 0.05$ & 0.009 \\
\hline
\end{tabular}

${ }^{\mathrm{a}}$ The $p$ values were calculated with paired Student's t-test. $p<0.05$ indicates that the difference between the compared parameter sets is statistically significant. This test has small compared data sets; ${ }^{b}$ Homogeneity Index $(\mathrm{HI})=\mathrm{D}_{5} / \mathrm{D}_{95}$; ${ }^{\mathrm{c}}$ Conformality Index $(\mathrm{CI})=\left(\mathrm{TV} \mathrm{RI}_{\mathrm{RI}} / \mathrm{TV}\right) \times\left(\mathrm{TV} \mathrm{RI}_{\mathrm{RI}} / \mathrm{V}_{\mathrm{RI}}\right)$, where $\mathrm{TV} \mathrm{RI}_{\mathrm{RI}}$ is the target volume covered by the prescription dose, TV is the target volume; $\mathrm{V}_{\mathrm{RI}}$ is the volume of the prescription dose. Ideal CI should be close to 1.0 [23].

Table 2. Normal tissue sparing.

\begin{tabular}{|c|c|c|c|c|c|}
\hline \multirow{2}{*}{ PTV } & \multicolumn{2}{|c|}{ Conventional 3D-CRT } & \multicolumn{2}{|c|}{ TomoDirect 3D-CRT } & \multirow{2}{*}{$p$ Value } \\
\hline & Range & Mean \pm SD & Range & Mean \pm SD & \\
\hline \multicolumn{6}{|c|}{ Heart } \\
\hline Mean Dose (Gy) & $0.83-1.74$ & $1.29 \pm 0.27$ & $0.55-1.24$ & $0.77 \pm 0.20$ & 0.000 \\
\hline $\mathrm{D}_{1 \mathrm{cc}}(\mathrm{Gy})$ & $3.56-33.16$ & $15.65 \pm 7.97$ & $1.93-27.52$ & $7.15 \pm 7.61$ & 0.000 \\
\hline $\mathrm{D}_{5}(\mathrm{~Gy})$ & $1.90-4.88$ & $3.08 \pm 0.81$ & $1.18-2.78$ & $1.75 \pm 0.45$ & 0.000 \\
\hline \multicolumn{6}{|l|}{ Ipsilateral Lung } \\
\hline Mean Dose (Gy) & $0.37-0.93$ & $0.67 \pm 0.16$ & $0.27-0.73$ & $0.42 \pm 0.14$ & 0.000 \\
\hline $\mathrm{D}_{1 \mathrm{cc}}(\mathrm{Gy})$ & $2.28-31.74$ & $15.16 \pm 11.29$ & $1.44-36.62$ & $10.38 \pm 12.16$ & 0.039 \\
\hline $\mathrm{D}_{5}(\mathrm{~Gy})$ & $1.04-2.55$ & $1.87 \pm 0.42$ & $0.67-1.98$ & $1.09 \pm 0.38$ & 0.000 \\
\hline \multicolumn{6}{|c|}{ Contralateral Lung } \\
\hline Mean Dose (Gy) & $0.12-0.30$ & $0.21 \pm 0.06$ & $0.13-0.28$ & $0.19 \pm 0.05$ & 0.015 \\
\hline $\mathrm{D}_{1 \mathrm{cc}}(\mathrm{Gy})$ & $0.66-1.45$ & $1.04 \pm 0.21$ & $0.52-1.07$ & $0.73 \pm 0.14$ & 0.000 \\
\hline $\mathrm{D}_{5}(\mathrm{~Gy})$ & $0.37-0.69$ & $0.56 \pm 0.08$ & $0.27-0.58$ & $0.39 \pm 0.08$ & 0.000 \\
\hline \multicolumn{6}{|c|}{ Contralateral Breast } \\
\hline Mean Dose (Gy) & $0.42-0.85$ & $0.59 \pm 0.12$ & $0.30-0.46$ & $0.39 \pm 0.05$ & 0.000 \\
\hline $\mathrm{D}_{\text {lcc }}(\mathrm{Gy})$ & $1.29-2.38$ & $1.91 \pm 0.32$ & $0.77-1.72$ & $1.29 \pm 0.32$ & 0.000 \\
\hline $\mathrm{D}_{5}(\mathrm{~Gy})$ & $0.90-1.58$ & $1.24 \pm 0.18$ & $0.55-0.98$ & $0.81 \pm 0.14$ & 0.000 \\
\hline \multicolumn{6}{|c|}{ Unspecified Tissue } \\
\hline Mean Dose (Gy) & $1.87-4.01$ & $3.00 \pm 0.69$ & $1.68-3.68$ & $2.28 \pm 0.53$ & 0.004 \\
\hline $\mathrm{D}_{\text {lcc }}(\mathrm{Gy})$ & $51.48-53.61$ & $52.54 \pm 0.66$ & $52.32-55.16$ & $53.74 \pm 0.83$ & 0.001 \\
\hline $\mathrm{D}_{5}(\mathrm{~Gy})$ & $7.46-40.14$ & $25.09 \pm 10.46$ & $2.90-40.04$ & $11.56 \pm 10.85$ & 0.004 \\
\hline
\end{tabular}




\section{Discussion}

Prone breast radiation treatment is the preferred technique at our facility since 2005 because it offers several potential advantages compared to supine patient positioning. With enhanced immobilization technique, treatment time and planning optimization can be improved with expected less toxicity with prone breast setup. Dosimetric studies have consistently demonstrated improved normal tissue toxicity with prone positioning, with clear dose reductions to the lung and potential benefits in dose to cardiac structures compared with supine treatment. Prone breast treatment for those patients with pendulant anatomy is critical in delivering uniform dosimetry to the whole breast volume. Though field-in-field prone breast planning has been implemented, however, with similar modality in Tomotherapy, it has achieved superior plans with better dose coverage in $\mathrm{D}_{95}$ and $\mathrm{D}_{99}$. Tomotherapy with TomoDirect also made the optimization process easier to accomplish the planning goals in eliminating hot spots. Prone positioning appears to be extremely well tolerated and reproducible. Finally, a recent report from our institution of the long-term clinical outcomes of prone whole breast treatment confirmed excellent tumor control parameters similar to that published with the conventional supine radiation therapy [13].

Table 1 has shown that with Tomotherapy planning, a better homogeneity and conformality indexes can be achieved the same time. This systematic comparison and the clinical finding can serve as the guidelines for further planning parameters selection with template implementation. As recommended in the Phase 3 NSABP B-39/ RTOG 0413 study, a comparison PTV, PTV_EVAL, was created to remove target volume outside the breast [19]. To allow comparison of normal structure radiation exposure in this series, treatment planning was performed to cover excellent PTV coverage $\left(\mathrm{V}_{95 \%}\right)$ with two techniques. For field-in-field and TomoDirect the coverage were calculated of $96.34 \%$ and $99.93 \%$, respectively. The critical structures such as heart, ipsilateral and contralateral lungs, contralateral breast, etc. all presented less mean doses in TD plans. Importantly, to achieve optimal PTV coverage, a much larger portion of normal tissue (higher integral dose) is required to receive radiation exposure for traditional helical Tomotherapy plans. This integral dose has been dramatically reduced to the level comparable to the LINAC prone breast plans with the TD software module. Whether this would lead to increased clinical complications remains to be seen with further follow-up.

This study allows a true dosimetric comparison of both modalities; field-in-field versus TD optimized plans, as the planning CT scans were performed in the same patient at same beam geometry. In the past, a significantly higher portion of ipsilateral breast tissue (to cover chest wall lymphatic nodes) needs to be irradiated to achieve higher target coverage with the supine external beam techniques, which is a risk of poor immobilization and repositioning problems on a daily basis. It is important to note that the clinical validity of using a prone Tomotherapy remains to be demonstrated and has to be studied in detail using setup reproducibility data. Tighter dosevolume constraints to PTV and normal tissues have been proposed and will be evaluated in ongoing trials to assess the feasibility and safety of this image guided therapy TD plan; thereby making MVCT a great pre-treatment verification in the clinical setting. The dosimetric benefits of TD and image verification for the positioning and planning approach must be tailored to the patient's anatomy and location of the lumpectomy cavity, which could be translated to homogeneous dose distribution and better cosmetic results.

\section{Conclusion}

A field-in-field planning approach with MLC combination is feasible for prone breast treatment and improves the dose homogeneity of those women with larger and pendulous breasts. With a different delivery platform, TD also provides another alternative by changing the delivery pattern with the ring gantry system and moving couch positions. We have evaluated and compared the dosimetry variation in two different planning modalities (3D field-in-field technique versus TomoDirect). Due to the planning and the delivery nature of $\mathrm{TD}$, the hot spot of the opposing beams can be minimized with better dose uniformity and conformity in the breast volume (PTV). The lung and heart with TD sparing can achieve better dosimetric results comparatively and the hot spots are also lower in dosimetric results. Contralateral breast dose is also minimized compared to the conventional 3D technique. Prone breast treatment in Tomotherapy is a choice for patients not suitable for the supine position, while at the same time like to achieve image guided verification with smooth and easier setup process. TD prone breast treatment means to minimize the patient positional uncertainties, and reduce the separation and cardiopulmonary irradiation. In $\mathrm{TD}$, more reduction in critical organ doses is also feasible by the modulated binary MLC delivery nature, with easier setup and image guided pretreatment verification. TD planning is relatively straightforward with carefully selected patient breast size and all those parameters can be properly screened during the virtual simulation stage. TD offers an innovative alternative to the current LINAC based prone breast treatment with optimum dosimetric outcomes.

\section{REFERENCES}

[1] L. Beloqui and J. M. Usategui, M. Clarke, R. Collins, S. 
Darby, C. Davies, P. Elphinstone, E. Evans, J. Goodwin, R. Gray, C. Hicks, C. James, E. MacKinnon, P. McGale, T. McHugh, R. Peto, C. Taylor and Y. Wang, "Effects of Radiotherapy and of Differences in the Extent of Surgery for Early Breast Cancer on Local Recurrence and 15-Year Survival: An Overview of the Randomized Trials," Lancet, Vol. 366, No. 9503, 2005, pp. 2087-2106.

[2] S. Pierce, A. Recht, T. Lingos and Noname, "Long-Term Radiation Complications Following Conservative Surgery (CS) and Radiation Therapy (RT) in Patients with Early Stage Breast Cancer," International Journal of Radiation Oncology*Biology*Physics, Vol. 23, No. 5, 1992, pp. 915-923. http://dx.doi.org/10.1016/0360-3016(92)90895-O

[3] L. Marks, X. Yu, R. Prosnitz, S. Zhou, P. Hardenbergy, M. Blazing, D. Hollis, P. Lind, A. Tisch, T. Wong and S. Borges-Neto, "The Incidence and Functional Consequences of RT-Associated Cardiac Perfusion Defects," International Journal of Radiation Oncology*Biology* Physics, Vol. 63, No. 1, 2005, pp. 214-223.

[4] S. Das, A. Baydush, S. Zhou, M. Miften, X. Yu, O. Craciunescu, M. Oldham, K. Light, T. Wong, M. Blazing, S. Borges-Neto, M. Dewhirst and L. Marks, "Predicting Radiotherapy-Induced Cardiac Perfusion Defects," Medical Physics, Vol. 32, No. 1, 2005, pp. 19-27. http://dx.doi.org/10.1118/1.1823571

[5] J. Gray, B. McCormick, L. Cox and J. Yahalom, "Primary Breast Irradiationin Large-Breasted or Heavy Women: Analysis of Cosmetic Outcome," International Journal of Radiation Oncology*Biology*Physics, Vol. 21, No. 2, 1991, pp. 347-354. http://dx.doi.org/10.1016/0360-3016(91)90781-X

[6] D. Clarke, A. Martinez and R. Cox, "Analysis of Cosmetic Results and Complications in Patients with Stage I and II Breast Cancer Treated by Biopsy and Irradiation," International Journal of Radiation Oncology*Biology*Physics, Vol. 9, No. 12, 1983, pp. 1807-1813.

http://dx.doi.org/10.1016/0360-3016(83)90348-6

[7] A. Grann, B. McCormick, E. Chabner, S. Gollamudi, K. Schupak, B. Mychalczak, A. Heerdt, T. Merchant and M. Hunt, "Prone Breast Radiotherapy in Early-Stage Breast Cancer: A Preliminary Analysis," International Journal of Radiation Oncology*Biology*Physics, Vol. 47, No. 2, 2000, pp. 319-325.

http://dx.doi.org/10.1016/S0360-3016(00)00448-X

[8] K. Goodman, L. Hong, R. Wagman, M. Hunt and B. McCormick, "Dosimetric Analysis of a Simplified Intensity Modulation Technique for Prone Breast Radiotherapy," International Journal of Radiation Oncology*Biology* Physics, Vol. 60, No. 1, 2004, pp. 95-102. http://dx.doi.org/10.1016/j.ijrobp.2004.02.016

[9] L. Kestin, M. Sharpe and R. Frazier, "Intensity Modulation to Improve Dose Uniformity with Tangential Breast Radiotherapy: Initial Clinical Experience," International Journal of Radiation Oncology*Biology* Physics, Vol. 48, No. 5, 2000, pp. 1559-1568. http://dx.doi.org/10.1016/S0360-3016(00)01396-1

[10] Y. Lo, G. Yasuda and T. Fitzgerald, "Intensity Modualiton for Breast Treatment Using Static Multi-Leaf Collimators," International Journal of Radiation Oncology*Biology*Phy- sics, Vol. 46, No. 1, 2000, pp. 187-194. http://dx.doi.org/10.1016/S0360-3016(99)00382-X

[11] B. Smith, I. Pan, Y. Shih, G. Smith, J. Harris, R. Punglia, L. Pierce, R. Jagsi, J. Hayman, S. Giordano and T. Buchholz, "Adoption of Intensity-Modulated Radiation Therapy for Breast Cancer in the United States," Journal of the National Cancer Institute, Vol. 103, No. 10, 2011, pp. 798-809. http://dx.doi.org/10.1093/jnci/djr100

[12] V. Croog, A. Wu, B. McCormick and K. Beal, “Accelerated Whole Breast Irradiation with Intensity-Modulated Radiotherapy to the Prone Breast," International Journal of Radiation Oncology*Biology*Physics, Vol. 73, No. 1, 2009, pp. 88-93.

http://dx.doi.org/10.1016/j.ijrobp.2008.04.036

[13] L. Stegman, K. Beal, M. Hunt, M. Fornier and B. McCormick, "Long-Term Clinical Outcomes of Whole-Breast Irradiation Delivered in the Prone Position," International Journal of Radiation Oncology*Biology*Physics, Vol. 68, No. 1, 2007, pp. 73-81.

[14] M. Alonso-Basanta, J. Ko, M. Badcock, K. DeWyngaert and S. Formenti, "Coverage of Axillary Lymph Nodes in Supine vs. Prone Breast Radiotherapy," International Journal of Radiation Oncology*Biology*Physics, Vol. 73, No. 3, 2009, pp. 745-751. http://dx.doi.org/10.1016/j.ijrobp.2008.04.040

[15] K. DeWyngaert, G. Jozsef, J. Mitchell, B. Rosenstein and S. Formenti, "Accelerated Intensity-Modulated Radiotherapy to Breast in Prone Position: Dosimetric Results," International Journal of Radiation Oncology*Biology* Physics, Vol. 68, No. 4, 2007, pp. 1251-1259. http://dx.doi.org/10.1016/j.ijrobp.2007.04.018

[16] B. Wen, H. Hsu, G. Formenti-Ujlaki, S. Lymberis, C. Magnolfi, X. Zhao, J. Chang, K. DeWyngaert, G. Jozsef and S. Formenti, "Prone Accelerated Partial Breast Irradiation after Breast-Conserving Surgery: Compliance to the Dosimetry Requirement of RTOG-0413," International Journal of Radiation Oncology*Biology* Physics, Vol. 84, No. 4, 2012, pp. 910-916. http://dx.doi.org/10.1016/j.ijrobp.2012.01.055

[17] C. Stella, J. Lymberis, K. DeWyngaert, A. Parhar, A. Chhabra, M. Fenton-Kerimian, J. Chang, T. Hochman, A. Guth, D. Roses, J. Goldberg and S. Formenti, "Prospective Assessemnt of Optimal Individual Position (Prone versus Supine) for Breast Radiotherapy: Volumetric and Dosimetric Correlation in 100 Patients," International Journal of Radiation Oncology*Biology*Physics, Vol. 84, No. 4, 2012, pp. 902-909. http://dx.doi.org/10.1016/j.ijrobp.2012.01.040

[18] S. Formenti, M. Truong, J. Goldberg, V. Mukhi, B. Rosenstein, D. Roses, R. Shapiro, A. Guth and K. DeWyngaert, "Prone Accelerated Partial Breast Irradiation after Breast-Conserving Surgery: Preliminary Clinical Resutls and Dose-Volume Histogram Analysis," International Journal of Radiation Oncology*Biology*Physics, Vol. 60, No. 2, 2004, pp. 493-504. http://dx.doi.org/10.1016/j.ijrobp.2004.04.036

[19] R. Patel, A. Becker, R. Das and T. Mackie, "A Dosimetric Comparison of Accelerated Partial Breast Irradiation Techniques: Multicatheter Interstitial Brachytherapy, Three- 
Dimensional Conformal Radiotherapy, and Supine versus Prone Helical Tomotherapy," International Journal of Radiation Oncology*Biology*Physics, Vol. 68, No. 3, 2007, pp. 935-942.

http://dx.doi.org/10.1016/j.ijrobp.2007.03.005

[20] A. Coon, A. Dickler, M. Kirk, Y. Liao, A. Shah, J. Strauss, S. Chen, J. Turian and K. Griem, "Tomotherapy and Multifield Intensity-Modulated Radiotherapy Planning Reduce Cardiac Doses in Left-Sided Breast Cancer Patients with Unfavorable Cardiac Anatomy," International Journal of Radiation Oncology*Biology*Physics, Vol. 78, No. 1, 2010, pp. 104-110. http://dx.doi.org/10.1016/j.ijrobp.2009.07.1705

[21] Y. Rong, T. Fahner and J. Welsh, "Hypofractionated Breast and Chest Wall Irradiation Using Simultaneous In-Field
Boost IMRT Delivered via Helical Tomotherapy," Technology in Cancer Research and Treatment, Vol. 7, No. 6, 2008, pp. 433-439.

[22] T. Reynders, K. Tournel, P. De Coninck, S. Heymann, V. Vinh-Hung, H. Van Parijs, M. Duchateau, N. Linthout, T. Gevaert, D. Verellen and G. Storme, "Dosimetric Assessment of Static and Helical Tomo Therapy in the Clinical Implementation of Breast Cancer Treatments," Radiotherapy \& Oncology, Vol. 93, No. 1, 2009, pp. 71-79. http://dx.doi.org/10.1016/j.radonc.2009.07.005

[23] L. Feuvret, G. Noel, J. Mazeron and P. Bey, "Conformity Index: A Review," International Journal of Radiation Oncology*Biology*Physics, Vol. 64, No. 2, 2006, pp. 333342. http://dx.doi.org/10.1016/j.ijrobp.2005.09.028 